\title{
Cardiovascular Morbidity in Psoriatic Arthritis: What Is the Effect of Inflammation?
}

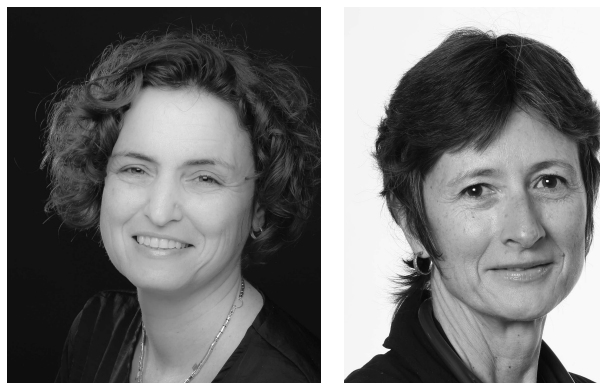

The association between chronic inflammatory arthritis and cardiovascular (CV) morbidity is well established. Patients with rheumatoid arthritis (RA), psoriatic arthritis (PsA), and ankylosing spondylitis are at increased risk for $\mathrm{CV}$ morbidity and mortality ${ }^{1}$. This risk remains high even after accounting for traditional CV risk factors, which highlights the potential role of chronic inflammation and its interaction with conventional risk factors in promoting atherosclerosis. However, much of the knowledge about $\mathrm{CV}$ comorbidities in rheumatic patients, its epidemiology, and its underlying mechanisms comes from studies in patients with RA, while such data are limited in PsA.

While some overlap can be found between PsA and RA in clinical features, treatment modalities, and underlying proinflammatory mechanisms, major differences exist between these 2 conditions. For example, PsA differs from RA in its demographics, the characteristics and extent of involvement of extraarticular tissues, the lack of autoantibodies, and the primary role that the interleukin (IL)-17/IL-23 pathway plays in disease evolution. These differences may affect CV risk and support the need for specific studies evaluating $\mathrm{CV}$ morbidity in patients with PsA. The study by Agca, et $a l^{2}$ in the current issue of The Journal assesses the effect of etanercept (ETN), an inhibitor of tumor necrosis factor- $\alpha$ (TNF- $\alpha$ ), on lipid levels and other CV risk factors in patients with PsA and thus addresses some of these gaps in knowledge.

What do we know about CV morbidity in PsA? It is accepted that the prevalence of CV disease (CVD) is elevated in patients with psoriasis and PsA compared with the general population. A recent metaanalysis found that $\mathrm{CV}$ morbidity is increased by $43 \%$ in patients with $\mathrm{PsA}^{3}$. The prevalence of established CV risk factors, such as metabolic syndrome, diabetes, hypertension, and dyslipidemia, is also elevated in psoriatic patients ${ }^{4}$, which raises the question as to whether these factors fully account for the elevated CV risk. Emerging data from epidemiologic studies suggest that part of this increased CV risk is unexplained by traditional risk factors, supporting the notion that PsA is an independent risk factor for $\mathrm{CV}$ events. This concept is supported by the strong link between the extent of psoriasis and PsA disease activity and various $\mathrm{CV}$ outcomes including soluble cardiometabolic biomarkers, burden of atherosclerotic plaques, and clinical CV events ${ }^{5,6}$.

The suppression of inflammation by immune-modulating agents may represent a promising new target for the lowering of CVD risk in patients with chronic inflammatory conditions because the ensuing benefits may apply beyond the skin and the joint disease ${ }^{7}$. In contrast to the strong evidence suggesting a potential beneficial effect of TNF- $\alpha$ inhibition on $\mathrm{CV}$ risk in patients with $\mathrm{RA}^{8}$, such information in psoriasis and PsA is limited and conflicting. Moreover, reliable data on the effect of TNF- $\alpha$ inhibitors on biomarkers of CVD in psoriatic patients are generally lacking. In their study, Agca, et al assessed 118 patients with PsA who initiated treatment with ETN over a period of up to 5 years. They evaluated the effect of TNF- $\alpha$ inhibition on lipid fractions and subfractions, and the association between PsA disease activity and $\mathrm{CV}$ risk factors. They report an increase in the levels of low-density lipoprotein cholesterol (LDL-C), high-density lipoprotein cholesterol (HDL-C), and triglycerides (TG) over 5 years following treatment initiation. Further, while total cholesterol (TC)/HDL-C ratio remained unchanged, apolipoprotein $\mathrm{B}$ to apolipoprotein AI ratio (ApoB/ApoAI) decreased, an observation that is of potential clinical significance because a higher ApoB/ApoAI ratio is associated with increased risk of fatal coronary events in the general population. These findings are in line with previous studies in RA that found an inverse association between lipid levels and disease activity in RA and an increase in lipids with effective antiinflammatory treatment. In a metaanalysis of 15 studies, treatment with TNF- $\alpha$ inhibitors was

See ETN in patients with PsA, page 1362

Personal non-commercial use only. The Journal of Rheumatology Copyright (C) 2017. All rights reserved. 
associated with an overall $10 \%$ increase in TC and a $7 \%$ increase in HDL-C within 6 months ${ }^{9}$. Smaller studies have shown increases in LDL-C and $\mathrm{ApoB}^{10}$. These seemingly unexpected phenomena were termed lipid paradox.

The lipid paradox refers to the observation that patients with active inflammation have relatively low levels of serum lipids, in direct contrast to the high $\mathrm{CV}$ risk associated with these rheumatic disorders. Further, successful antiinflammatory treatment, which is associated with reduction in $\mathrm{CV}$ risk, is often linked with subsequent elevation in serum lipid fractions, as described in the current study ${ }^{2}$ and in other rheumatic conditions, particularly in RA.

What are some of the mechanisms explaining the lipid paradox? The interactions between inflammation and lipid metabolism driving the lipid paradox phenomenon are complex and not entirely clear. However, inflammatory states are associated with high lipid demands to maintain energy and substrate homeostasis required to support effective function of the immune system (e.g., cell division, protein and hormone synthesis, tissue repair). Thus, the reduction in lipid levels observed during periods of active inflammation may be more a consequence of relative increased rate of catabolism of lipoproteins than reduced production. This is supported by the observation that infusion of proinflammatory cytokines such as TNF- $\alpha$ and IL-6 leads to lowering of TC, HDL-C, and LDL-C serum levels and increased uptake of LDL-C by hepatocytes ${ }^{11,12}$. Inflammation may also result in changes in serum concentration, composition, and properties of lipoprotein and composite subfractions that are not detected using standard lipid analytic techniques. Some of the observed changes include increases in the proatherogenic small dense and highly oxidized LDL-C particles and in lipoprotein (a) $[\mathrm{Lp}(\mathrm{a})]$, a type of LDL that is an independent risk factor for CVD. A proinflammatory milieu may also contribute to attenuation of the cardioprotective properties of HDL-C through relative lowering of the potent HDL-2 subfraction while also increasing "proinflammatory" HDL-C, with consequent functional impairment of the HDL particles regarding antioxidant, antiinflammatory, and reverse cholesterol capacities ${ }^{13,14,15}$.

The study by Agca, et al provides important information about the interplay between inflammation and lipid concentrations in PsA. The increase in levels of TC, LDL-C, HDL-C, and TG following TNF- $\alpha$ inhibition has been reported in a number of small studies in patients with psoriasis and PsA ${ }^{16,17}$. The strengths of the present study ${ }^{2}$ are its relatively large sample size and the long followup period of 5 years. In addition, the assessment of lipid subparticles, including ApoAI and ApoB is an important strength of this study. The reduction in ApoB/ApoAI ratio, which has been associated with a favorable CV outcome in the general population in the setting of a general increase in lipids levels, highlights the complexity of interpreting changes in conventional lipid profile in the context of active inflammation and antiinflammatory therapy. In line with this finding, others have reported potentially favorable changes in lipid particles, such as a reduction in $\mathrm{Lp}$ (a) and an increase in ApoAI, following TNF- $\alpha$ inhibitors in patients with PsA ${ }^{18}$. A similar phenomenon was observed in RA with other biologic therapies including IL-6 inhibitors and Janus kinase inhibitors ${ }^{19,20}$. However, it is important to keep in mind that although lipid changes observed in patients following the successful antiinflammatory treatment may reflect normalization of an "inflammatory dyslipidemia," as noted by the authors, limited data exist about the effect of TNF- $\alpha$ inhibition on hard clinical outcomes including $\mathrm{CV}$ events and mortality in patients with psoriatic disease. Other important gaps in knowledge include the effect of newer medications for psoriatic disease, including PDE4 inhibitors, IL-17 inhibitors, and IL-23 inhibitors, on CV outcomes. It is unclear whether suppression of psoriasis and PsA disease activity, regardless of the mode of therapy, underlies the changes observed in lipid profile or that other mechanisms specific to each biologic therapy influence the levels and pattern of lipid variables.

An obvious strategy to reduce $\mathrm{CV}$ risk in patients with PsA is to identify and optimize CV risk factors, including dyslipidemia. After all, this patient population is known to have a high prevalence of metabolic syndrome and related adverse CV risk profiles. However, although this approach may appear simple, it involves many complexities. What, then, is the optimal approach for managing dyslipidemia in patients with PsA? Based on the results of this study and others, clinicians are advised to assess lipid profile when disease activity is stable because the level of inflammation and treatment modifications may be associated with fluctuations in serum lipid fractions and subfractions. Elevation of components of the lipid profile could be expected after initiation of TNF- $\alpha$ inhibitors and potentially other antiinflammatory medications. However, because the levels of TC, LDL-C, and HDL-C tend to increase in parallel, incorporating lipid levels into clinical prediction algorithms (e.g., Framingham risk score) will usually result in minimal modification in the estimated CV risk. However, the risk prediction tools do not currently include inflammatory joint disease in the risk assessment algorithms, leading to potential underestimation of risk in patients with PsA. Additional studies are required to better understand the link between inflammatory pathways in psoriatic disease and lipid metabolism, their effect on CV outcomes and implications for assessment, and targeted risk factor interventions to improve $\mathrm{CV}$ prevention strategies in psoriatic patients.

LIHI EDER, MD, PhD; PAULA HARVEY, BMBS, PhD, Women's College Research Institute, Women's College Hospital, and Department of Medicine, University of Toronto, Toronto, Ontario, Canada.

Personal non-commercial use only. The Journal of Rheumatology Copyright @ 2017 . All rights reserved. 
Address correspondence to Dr. L. Eder, Toronto Western Hospital, Centre for Prognosis Studies in The Rheumatic Diseases, 399 Bathurst St., Toronto, Ontario M5T 2S8, Canada.E-mail: leder@uhnresearch.ca, benlihi@gmail.com

\section{REFERENCES}

1. Schieir O, Tosevski C, Glazier RH, Hogg-Johnson S, Badley EM. Incident myocardial infarction associated with major types of arthritis in the general population: a systematic review and meta-analysis. Ann Rheum Dis 2017 Feb 20 (E-pub ahead of print).

2. Agca R, Heslinga M, Kneepkens EL, van Dongen C, Nurmohamed MT. The effects of 5-year etanercept therapy on cardiovascular risk factors in patients with psoriatic arthritis. J Rheumatol 2017; 44:1362-8.

3. Polachek A, Touma Z, Anderson M, Eder L. Risk of cardiovascular morbidity in patients with psoriatic arthritis: a meta-analysis of observational studies. Arthritis Care Res 2017;69:67-74.

4. Miller IM, Ellervik C, Yazdanyar S, Jemec GB. Meta-analysis of psoriasis, cardiovascular disease, and associated risk factors. J Am Acad Dermatol 2013;69:1014-24.

5. Eder L, Thavaneswaran A, Chandran V, Cook R, Gladman DD. Increased burden of inflammation over time is associated with the extent of atherosclerotic plaques in patients with psoriatic arthritis. Ann Rheum Dis 2015;74:1830-5.

6. Eder L, Wu Y, Chandran V, Cook R, Gladman DD. Incidence and predictors for cardiovascular events in patients with psoriatic arthritis. Ann Rheum Dis 2016;75:1680-6.

7. Ridker PM. Moving beyond JUPITER: will inhibiting inflammation reduce vascular event rates? Curr Atheroscler Rep 2013;15:295.

8. Roubille C, Richer V, Starnino T, McCourt C, McFarlane A, Fleming $\mathrm{P}$, et al. The effects of tumour necrosis factor inhibitors, methotrexate, non-steroidal anti-inflammatory drugs and corticosteroids on cardiovascular events in rheumatoid arthritis, psoriasis and psoriatic arthritis: a systematic review and meta-analysis. Ann Rheum Dis 2015;74:480-9.

9. van Sijl AM, Peters MJ, Knol DL, de Vet RH, Sattar N, Dijkmans BA, et al. The effect of TNF-alpha blocking therapy on lipid levels in rheumatoid arthritis: a meta-analysis. Semin Arthritis Rheum 2011;41:393-400.

10. Hassan S, Milman U, Feld J, Eder L, Lavi I, Cohen S, et al. Effects of anti-TNF- $\alpha$ treatment on lipid profile in rheumatic diseases: an analytical cohort study. Arthritis Res Ther 2016;18:261.

11. Khovidhunkit W, Kim MS, Memon RA, Shigenaga JK, Moser AH, Feingold KR, et al. Effects of infection and inflammation on lipid and lipoprotein metabolism: mechanisms and consequences to the host. J Lipid Res 2004:45:1169-96.

12. Ettinger WH, Varma VK, Sorci-Thomas M, Parks JS, Sigmon RC, Smith TK, et al. Cytokines decrease apolipoprotein accumulation in medium from Hep G2 cells. Arterioscler Thromb 1994;14:8-13.

13. Wang J, Hu B, Kong L, Cai H, Zhang C. Native, oxidized lipoprotein(a) and lipoprotein(a) immune complex in patients with active and inactive rheumatoid arthritis: plasma concentrations and relationship to inflammation. Clin Chim Acta 2008;390:67-71.

14. Mehta NN, Li R, Krishnamoorthy P, Yu Y, Farver W, Rodrigues A, et al. Abnormal lipoprotein particles and cholesterol efflux capacity in patients with psoriasis. Atherosclerosis 2012;224:218-21.

15. Hashizume M, Mihara M. Atherogenic effects of TNF- $\alpha$ and IL-6 via up-regulation of scavenger receptors. Cytokine 2012;58:424-30

16. Lestre S, Diamantino F, Veloso L, Fidalgo A, Ferreira A. Effects of etanercept treatment on lipid profile in patients with moderate-to-severe chronic plaque psoriasis: a retrospective cohort study. Eur J Dermatol 2011;21:916-20.

17. Maneiro JR, Souto A, Gomez-Reino JJ. Impact of treatment with TNF antagonists on total cholesterol in patients with ankylosing spondylitis and psoriatic arthritis. Clin Rheumatol 2017;36:1167-72.

18. Sattar N, Crompton P, Cherry L, Kane D, Lowe G, McInnes IB. Effects of tumor necrosis factor blockade on cardiovascular risk factors in psoriatic arthritis: a double-blind, placebo-controlled study. Arthritis Rheum 2007;56:831-9.

19. Daïen CI, Duny Y, Barnetche T, Daures JP, Combe B, Morel J. Effect of TNF inhibitors on lipid profile in rheumatoid arthritis: a systematic review with meta-analysis. Ann Rheum Dis 2012;71:862-8.

20. Souto A, Salgado E, Maneiro JR, Mera A, Carmona L, Gómez-Reino JJ. Lipid profile changes in patients with chronic inflammatory arthritis treated with biologic agents and tofacitinib in randomized clinical trials: a systematic review and meta-analysis. Arthritis Rheum 2015;67:117-27.

J Rheumatol 2017;44:1295-7; doi:10.3899/jrheum.170534 